\title{
Speed Estimation Direct Stator Field-orientation-controlled Induction Motor Drive Using Adaptive Flux Estimator
}

\author{
Yung-Chang Luo, ${ }^{*}$ Bo-Wei Chen, Wen-Cheng Pu, and Neng-Sheng Pai \\ Department of Electrical Engineering, National Chin-Yi University of Technology, \\ No. 57, Sec. 2, Zhongshan Rd, Taiping Dist, Taichung 41170, Taiwan (ROC)
}

(Received July 23, 2019; accepted October 4, 2019)

Keywords: speed estimation, flux estimator, current sensor, fuzzy logic control (FLC) strategy, model reference adaptive system (MRAS)

In this study, a speed estimation scheme using the fuzzy logic control (FLC) strategy adaptive flux estimator is presented for a direct stator field-orientation-controlled (DSFOC) induction motor (IM) drive. The current and flux of the stator were utilized to establish the DSFOC IM drive, and the stator current was obtained from the IM by utilizing Hall effect current sensors. The adaptive stator flux estimator was developed according to the voltagemodel and current-model stator flux estimators, and the estimated rotor speed was derived from this flux estimator. The adaptation mechanism of the adaptive stator flux estimator was designed using the FLC strategy. The synchronous position angle for the coordinate transformation between the stationary and synchronous reference coordinate frames was developed using the model reference adaptive system (MRAS) based on the reactive power of an IM. The MATLAB $\backslash$ Simulink ${ }^{\circledR}$ toolbox was used to simulate this system, and all the control algorithms were realized using a 32-bit RX62T microcontroller to validate this approach. Both simulation and experimental results (including the estimated rotor speed, electromagnetic torque, and stator flux locus) confirmed the effectiveness of the proposed system and validated the proposed approach.

\section{Introduction}

Intelligent production is a trend in modern industry, while motors, in general, are essential actuators for tool machines. Induction motors (IMs) are popularly adopted because of their reliability, robustness, low cost, and low maintenance. However, the mathematical model of an IM is nonlinear, time-variant, and coupled, making the control of an IM drive more difficult than that of a DC motor drive. According to the field orientation controlled (FOC) theory of the IM, ${ }^{(1)}$ by coordinate transformation, the three-phase alternating current signals of an IM can be transformed into the torque- and flux-current components, both of which are orthogonal and can be separately dominated. This condition is analogous to a separately excited DC motor, and the maximum torque-to-current ratio is attained. The implementation of a conventional FOC

*Corresponding author: e-mail: luoyc@ncut.edu.tw https://doi.org/10.18494/SAM.2020.2574 
IM drive requires a shaft position sensor, such as an encoder or a resolver, to detect the rotorshaft position. This position sensor, however, reduces the drive robustness and is not suitable for such a hostile environment. Hence, the development of the speed estimation FOC IM drive is necessary to replace the conventional one. According to the literature, some estimation methods for the speed estimation FOC IM drive have been proposed: speed identification derived from an adaptive control system, ${ }^{(2-4)}$ speed estimation in accordance with a neural network or fuzzy logic control (FLC) approach, ${ }^{(5-8)}$ speed adjustment by a flux estimator or a flux observer, ${ }^{(9-12)}$ and speed determination from an extended Kalman filter. ${ }^{(13-15)}$

The direct stator FOC (DSFOC) IM drive, by the application of the measured voltages and currents of the motor, directly estimates the angle and magnitude of the stator flux position. However, the indirect stator FOC (SFOC) IM drive uses the estimated slip angle and rotor position to calculate the stator flux position. A comparison of the DSFOC type with the indirect SFOC type shows that the DSFOC type is unaffected by the rotor time constant. ${ }^{(16)}$ In this study, we utilized the model reference adaptive system (MRAS) to develop both the adaptive stator flux estimator and the adaptive estimated synchronous position angle scheme. The adaptive stator flux estimator was developed on the basis of the voltage-model and currentmodel stator flux estimators, and the estimated rotor speed was derived from this adaptive stator flux estimator. The adjustment mechanism of the adaptive stator flux estimator was designed using the FLC strategy. Furthermore, the reactive power of an IM was utilized to establish an adaptive estimated synchronous position angle scheme; this estimated synchronous position angle guaranteed that the coordinate transformation between the stationary and synchronous reference coordinate frames would be exact. Here, the stator current was acquired from an IM by utilizing Hall effect current sensors. These approaches guaranteed the achievement of the perfect speed estimation DSFOC IM drive.

This paper has five sections. In Sect. 1, research motivation, background, and a review of the literature on speed estimation methods for FOC IM drives are presented. The decoupled DSFOC IM drive system and linear controller design are covered in Sect. 2. The MRAS synchronous position angle identification for the realization of the exact coordinate transformation is described in Sect. 3. The adaptive speed estimation scheme using the FLC strategy stator flux estimator is described in Sect. 4. The simulation and experiment are discussed in Sect. 5.

\section{DSFOC IM Drive}

The state equations of an IM expressed as the current and flux of the stator at the synchronous reference coordinate frame are given by

$$
\begin{gathered}
p \vec{i}_{s}^{e}=-\left(\frac{R_{s}}{L_{\sigma}}+\frac{1}{\sigma \tau_{r}}+j \omega_{s l}\right) \vec{i}_{s}^{e}+\frac{1}{L_{\sigma}}\left(\frac{1}{\tau_{r}}-j \omega_{r}\right) \vec{\lambda}_{s}^{e}+\frac{1}{L_{\sigma}} \vec{v}_{s}^{e}, \\
p \vec{\lambda}_{s}^{e}=-R_{s} \vec{i}_{s}^{e}-j \omega_{e} \vec{\lambda}_{s}^{e}+\vec{v}_{s}^{e},
\end{gathered}
$$


where $j$ is the imaginary part and $R_{S}$ and $R_{r}$ are the stator and rotor resistances, respectively. $L_{S}$, $L_{r}$, and $L_{m}$ are the stator, rotor, and mutual inductances, respectively, $\sigma=1-\left(L_{m}^{2} / L_{s} L_{r}\right)$ is the leakage inductance coefficient, $L_{\sigma}=\sigma L_{s}$ is the stator leakage inductance, and $\tau_{r}=L_{r} / R_{r}$ is the rotor time constant. $\vec{i}_{s}^{e}=i_{d s}^{e}+j i_{q s}^{e}, \vec{v}_{s}^{e}=v_{d s}^{e}+j v_{q s}^{e}$, and $\vec{\lambda}_{s}^{e}=\lambda_{d s}^{e}+j \lambda_{q s}^{e}$ are the current, voltage, and flux of the stator, respectively, $\omega_{e}$ is the speed of the synchronous reference coordinate frame, $\omega_{r}$ is the electric speed of the rotor, $\omega_{s l}=\omega_{e}-\omega_{r}$ is the slip speed, and $p=d / d t$ is the differential operator.

Under an SFOC condition, setting $\lambda_{q s}^{e}=0$ in Eq. (1), the estimated slip speed and $d$-axis stator flux are respectively derived as

$$
\begin{gathered}
\hat{\omega}_{s l}=\frac{\left(1+\sigma \tau_{r} s\right) L_{s} i_{q s}^{e}}{\tau_{r}\left(\lambda_{d s}^{e}-\sigma L_{s} i_{d s}^{e}\right)}, \\
\frac{1+\tau_{r} s}{\left(1+\sigma \tau_{r} s\right) L_{s}} \hat{\lambda}_{d s}^{e}=i_{d s}^{e}-\frac{\sigma \tau_{r} \hat{\omega}_{s l}}{1+\sigma \tau_{r} s} i_{q s}^{e},
\end{gathered}
$$

where "^" implies an estimated value and $s$ is the Laplace operator. The examination of Eq. (4) shows that the second term on the right side is a coupling component in relation to the $q$-axis stator current. This coupling component allows the definition of the $d$-axis stator current feedforward compensation as

$$
i_{d s_{-} c o m p}^{e}=\frac{\sigma \tau_{r} \hat{\omega}_{s l}}{1+\sigma \tau_{r} s} i_{q s}^{e}
$$

This compensation $i_{d s_{-}}^{e}$ comp will be placed on the output of the $d$-axis stator current control loop. Hence, the linear control relationship between the estimated $d$-axis stator flux and the $d$-axis stator current is given by

$$
\hat{\lambda}_{d s}^{e}=\frac{\left(1+\sigma \tau_{r} s\right) L_{s}}{1+\tau_{r} s} i_{d s}^{e}
$$

Under an SFOC condition, the developed electromagnetic torque of an IM can be derived as

$$
T_{e}=\frac{3 P}{4} \hat{\lambda}_{d s}^{e} i_{q s}^{e}
$$

where $P$ is the number of motor poles. Both $\hat{\lambda}_{d s}^{e}$ and $i_{q s}^{e}$ are orthogonal and can be independently controlled, as shown in Eq. (7), so that the maximum torque-to-current ratio of an IM is achieved. The mechanical equation of the motor is expressed as 


$$
J_{m} p \omega_{r m}+B_{m} \omega_{r m}+T_{L}=T_{e}
$$

where $J_{m}$ is the inertia of the motor, $B_{m}$ is the viscous friction coefficient, $T_{L}$ is the load torque, and $\omega_{r m}=(2 / P) \omega_{r m}$ is the mechanical speed of the motor shaft.

Under an SFOC condition, according to Eq. (2), the $d$-axis and $q$-axis stator voltage equations are respectively given by

$$
\begin{gathered}
R_{s} i_{d s}^{e}+p \hat{\lambda}_{d s}^{e}=v_{d s}^{e}, \\
R_{s} i_{q s}^{e}+\omega_{e} \hat{\lambda}_{d s}^{e}=v_{q s}^{e} .
\end{gathered}
$$

The examination of Eq. (9) shows that the linear relationship of the $d$-axis stator current control loop is inherent. The examination of Eq. (10) shows that the second term on the left side is a coupling component in relation to the $d$-axis stator voltage. By definition, the $q$-axis stator voltage feed-forward compensation is expressed as

$$
v_{q s_{-} \text {comp }}^{e}=\omega_{e} \hat{\lambda}_{d s}^{e}
$$

Thus, the linear relationship of the $q$-axis stator current control loop is achieved. The voltage commands of the $d$-axis and $q$-axis stator current control loops are respectively acquired as

$$
\begin{gathered}
v_{d s}^{e^{*}}=v_{d s}^{e^{\prime}}, \\
v_{q s}^{e^{*}}=v_{q s}^{e^{\prime}}+v_{q s_{-} c o m p}^{e},
\end{gathered}
$$

where $v_{d s}^{e^{\prime}}$ and $v_{q s}^{e^{\prime}}$ are the outputs of the $d$-axis and $q$-axis stator current controllers, respectively.

The linear control block diagram of an IM based on the DSFOC condition is shown in Fig. 1. Here, $K_{p d}$ and $K_{i d}$ are the proportional and integral gains of the $d$-axis stator current controller, $K_{p q}$ and $K_{i q}$ are the proportional and integral gains of the $q$-axis stator current controller, $K_{p f}$ and $K_{i f}$ are the proportional and integral gains of the flux controller, $K_{p s}$ and $K_{i s}$ are the proportional and integral gains of the speed controller, respectively. ${ }^{(16)}$

\section{MRAS Synchronous Position Angle Identification Based on Reactive Power}

A perfect DSFOC IM drive requires the exact synchronous position angle for the implementation of the coordinate transformation between the synchronous and stationary reference coordinate frames. In this system, the synchronous position angle was derived from the developed MRAS based on the reactive power of an IM. 


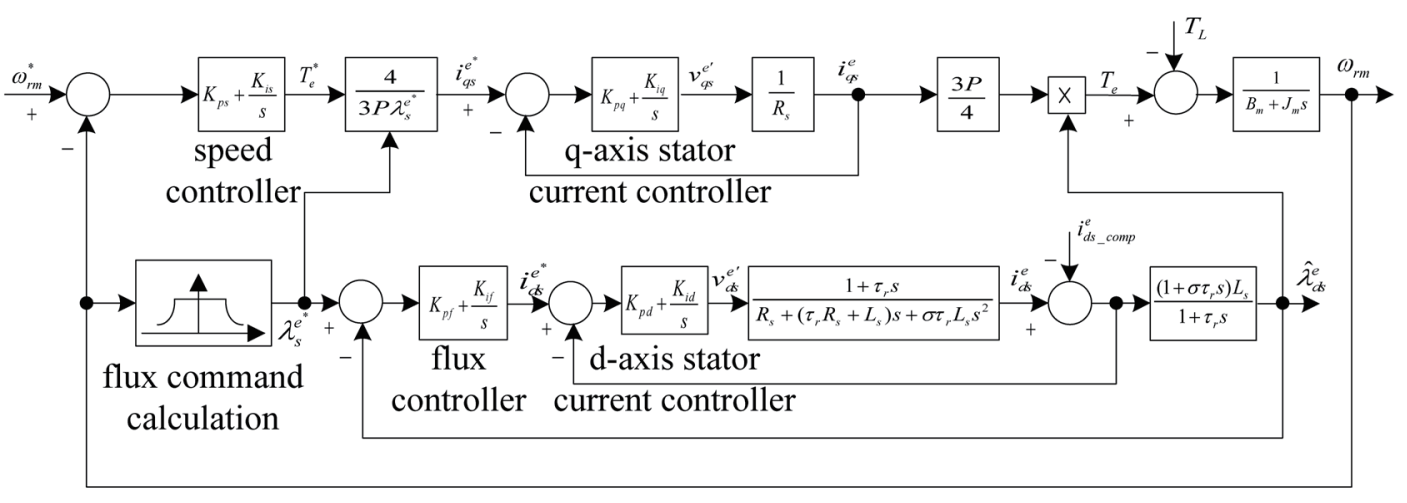

Fig. 1. Block diagram of linear control DSFOC IM drive.

According to Eq. (2), the $d$-axis and $q$-axis stator voltages respectively come from

$$
\begin{aligned}
& v_{d s}^{e}=R_{s} i_{d s}^{e}+p \lambda_{d s}^{e}-\omega_{e} \lambda_{q s}^{e}, \\
& v_{q s}^{e}=R_{s} i_{q s}^{e}+p \lambda_{q s}^{e}+\omega_{e} \lambda_{d s}^{e} .
\end{aligned}
$$

The reactive power of an IM acquired from the power source is given by

$$
Q=v_{q s}^{e} i_{d s}^{e}-v_{d s}^{e} i_{q s}^{e}
$$

Under an SFOC condition, by substituting Eqs. (14) and (15) into Eq. (16) and setting $\lambda_{q s}^{e}=0$, the reactive power of an IM can also be expressed as

$$
Q^{\prime}=\hat{\omega}_{e} i_{d s}^{e} \hat{\lambda}_{d s}^{e}-i_{q s}^{e} p \hat{\lambda}_{d s}^{e}
$$

According to the MRAS theory, ${ }^{(17)}$ Eq. (16) is selected as the reference model because it does not include the estimated variable $\hat{\omega}_{e}$, and Eq. (17) contains $\hat{\omega}_{e}$, and is selected as the adjustable model. The difference between the reference model and the adjustable model is applied to an adaptation mechanism to tune the estimated synchronous speed $\hat{\omega}_{e}$. The estimated synchronous position angle $\hat{\theta}_{e}$ for the implementation of the coordinate transformation between the synchronous and stationary reference coordinate frames is acquired by using $\hat{\omega}_{e}$ via an integrator $1 / s$. The proposed MRAS synchronous position angle identification scheme, based on the reactive power, is shown in Fig. 2. Here, a proportional-integral (P-I)-type adaptation mechanism was used to promote the response of the estimated synchronous position angle. 


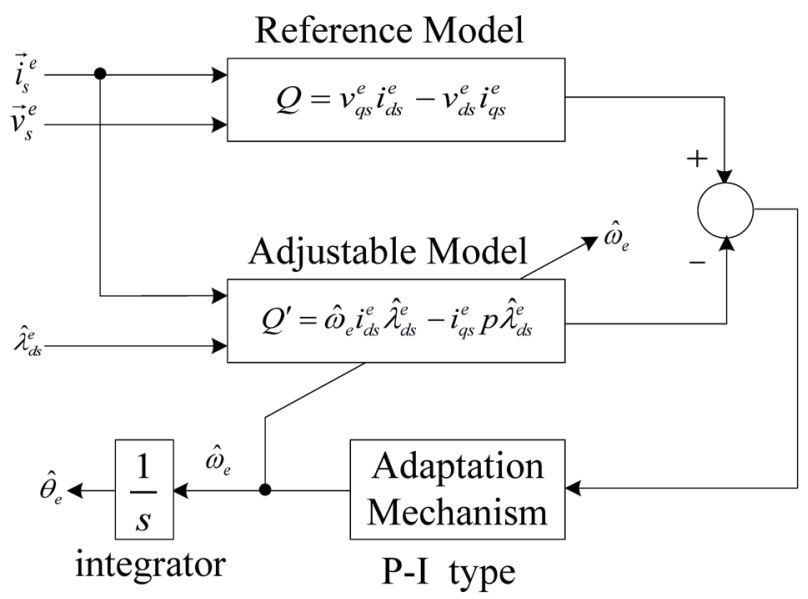

Fig. 2. MRAS synchronous position angle identification scheme based on reactive power.

\section{Speed Estimation Scheme of DSFOC IM Drive}

The feedback speed is replaced by a speed estimation signal in the speed estimation DSFOC IM drive, and this speed estimation signal is derived from the designed adaptive stator flux estimator.

\subsection{Adaptive speed estimation scheme based on stator flux estimator}

The stator and rotor voltage equations of an IM at the stationary reference coordinate frame $\left(\omega_{e}=0\right)$ can also be given by ${ }^{(16)}$

$$
\begin{gathered}
R_{s} \vec{i}_{s}^{s}+p \vec{\lambda}_{s}^{s}=\vec{v}_{s}^{s}, \\
R_{r} \vec{i}_{r}^{s}-j \omega_{r} \vec{\lambda}_{r}^{s}+p \vec{\lambda}_{r}^{s}=0,
\end{gathered}
$$

where $\vec{i}_{r}^{s}=i_{d r}^{s}+j i_{q r}^{s}$ and $\vec{\lambda}_{r}^{s}=\lambda_{d r}^{s}+j \lambda_{q r}^{s}$ are the current and flux of the rotor, respectively. The stator and rotor fluxes, are also respectively given by

$$
\begin{aligned}
& \vec{\lambda}_{s}^{s}=L_{s} \vec{i}_{s}^{s}+L_{m} \vec{i}_{r}^{s}, \\
& \vec{\lambda}_{r}^{s}=L_{r} \vec{i}_{r}^{s}+L_{m} \vec{i}_{s}^{s} .
\end{aligned}
$$

According to Eq. (18), the voltage-model stator flux estimator is developed as

$$
\hat{\vec{\lambda}}_{s v}^{s}=\frac{1}{s} \cdot\left(\vec{v}_{s}^{s}-R_{s} \vec{i}_{s}^{s}\right) .
$$


By utilizing Eqs. (19) and (21), the rotor flux can be expressed as

$$
\vec{\lambda}_{r}^{s}=\frac{L_{m} / \tau_{r}}{s+\left(1 / \tau_{r}-j \omega_{r}\right)} \vec{i}_{s}^{s}
$$

and by utilizing Eqs. (20) and (21), the stator flux is given by

$$
\vec{\lambda}_{s}^{s}=L_{\sigma} \vec{i}_{s}^{s}+\frac{L_{m}}{L_{r}} \vec{\lambda}_{r}^{s}
$$

By substituting Eq. (23) into Eq. (24), the current-model stator flux estimator is developed as

$$
\hat{\bar{\lambda}}_{s i}^{s}=L_{\sigma} \vec{i}_{s}^{s}+\frac{L_{m}}{L_{r}} \cdot \frac{L_{m} / \tau_{r}}{s+1 / \tau_{r}-j \hat{\omega}_{r}} \vec{i}_{s}^{s}
$$

The examination of the current-model stator flux estimator shows that it contains the estimated rotor speed $\hat{\omega}_{r}$, while the examination of the voltage-model stator flux estimator shows that it does not contain $\hat{\omega}_{r}$. According to the MRAS theory, ${ }^{(17)}$ Eqs. (22) and (25) are selected as the reference and adjustable models, respectively. The difference between the reference model and the adjustable model is expressed in Eq. (26), and this difference is applied to an adaptation mechanism to adjust the estimated rotor speed $\hat{\omega}_{r .}{ }^{(18)}$

$$
\varepsilon=\hat{\lambda}_{d s i}^{s} \hat{\lambda}_{q s v}^{s}-\hat{\lambda}_{q s i}^{s} \hat{\lambda}_{d s s}^{s}
$$

The proposed MRAS estimated rotor speed scheme based on the voltage-model and currentmodel stator flux estimators is shown in Fig. 3. Here, the adaptation mechanism was designed using the FLC strategy.

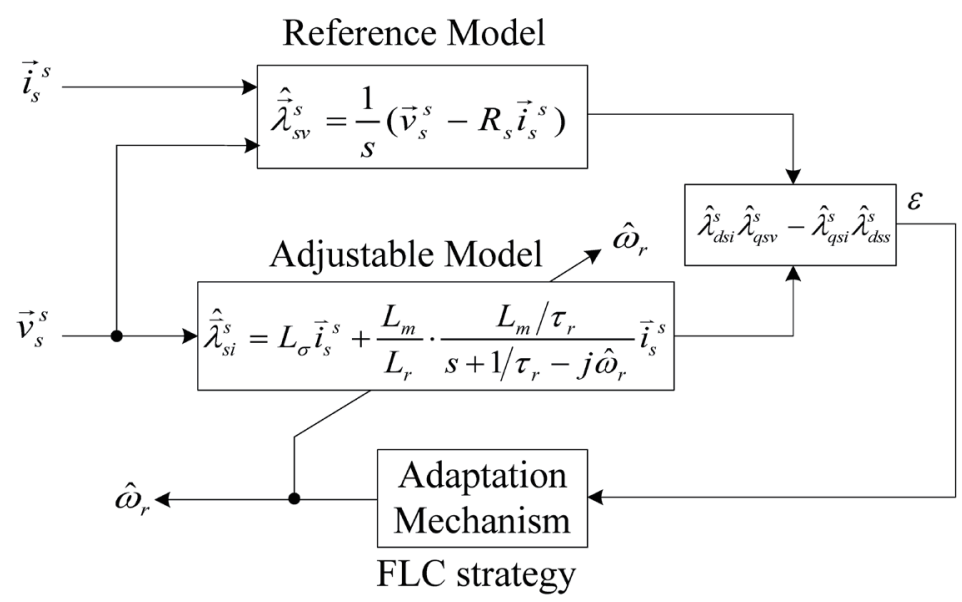

Fig. 3. MRAS estimated rotor speed based on stator flux estimator. 


\subsection{FLC adaptation mechanism design}

The FLC strategy was used to design the adaptation mechanism of the MRAS rotor speed identification scheme for the speed estimation DSFOC IM drive because it is simple, easy to implement, and requires no precise information about the mathematical model. ${ }^{(19)}$ The FLC applies the linguistic, imprecise knowledge of human experts and the behavioral characteristics of the plant. According to the linguistic rule, the definite quantification input signals including the model difference and its derivative via fuzzy inference decide the fuzzy output signals; these signals are then transformed to definite quantities for the estimation of the rotor speed. The FLC adaptation mechanism includes fuzzification, a fuzzy knowledge base, fuzzy inference, and defuzzification. ${ }^{(20)}$ The fuzzy knowledge base also has a fuzzy set and a rule base, as shown in Fig. 4. The model difference $\varepsilon$ and its derivative $\dot{\varepsilon}$ are input variables, the estimated rotor speed $\hat{\omega}_{r}$ is the output variable, $K_{1}$ and $K_{2}$ are the input scaling factors, and $K_{3}$ is the output scaling factor.

The design flow chart of an FLC adaptation mechanism is shown in Fig. 5, including the definition of the definite input and output variables, fuzzification and membership function, control rules and fuzzy inference, and defuzzification.

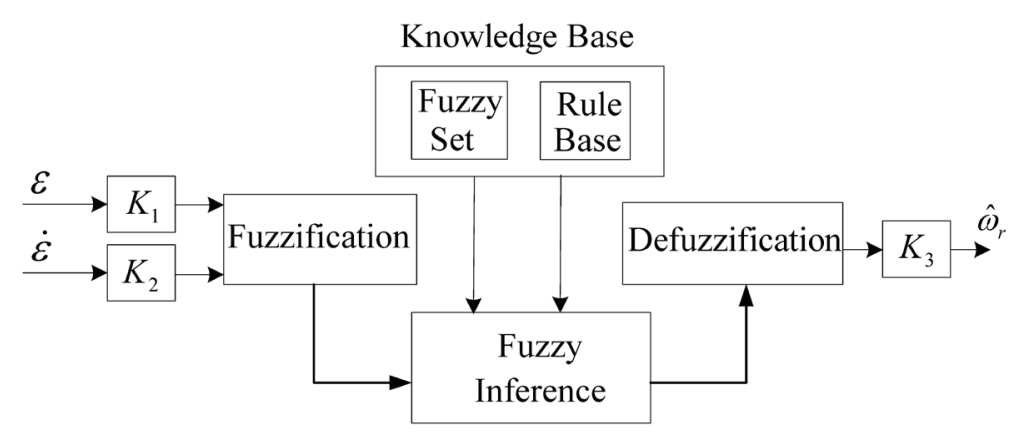

Fig. 4. FLC adaptation mechanism.

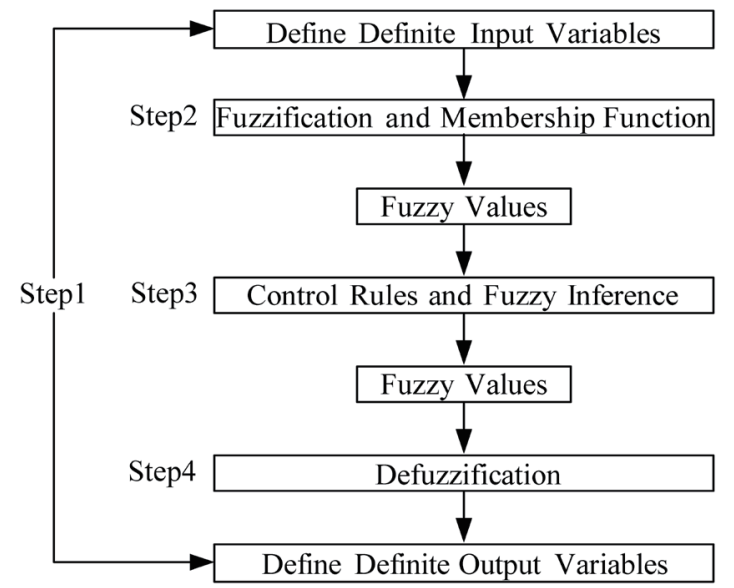

Fig. 5. Design flow chart of an FLC adaptation mechanism. 


\subsubsection{Fuzzification}

According to the fuzzy set theory, the fuzzification function converts crisp input values into corresponding fuzzy values, and the number of fuzzy sets determines the output of the adaptation mechanism. In this system, the fuzzy set is defined as shown in Table 1. The membership functions for the model difference $(\varepsilon)$, the derivative of the model difference $(\dot{\varepsilon})$, and the estimated rotor speed $\left(\hat{\omega}_{r}\right)$ are shown in Figs. 6-8, respectively.

Table 1

Definitions of fuzzy set.

\begin{tabular}{ll}
\hline NL & negative large \\
NS & negative small \\
ZE & zero error \\
PS & positive small \\
PL & positive large \\
\hline
\end{tabular}

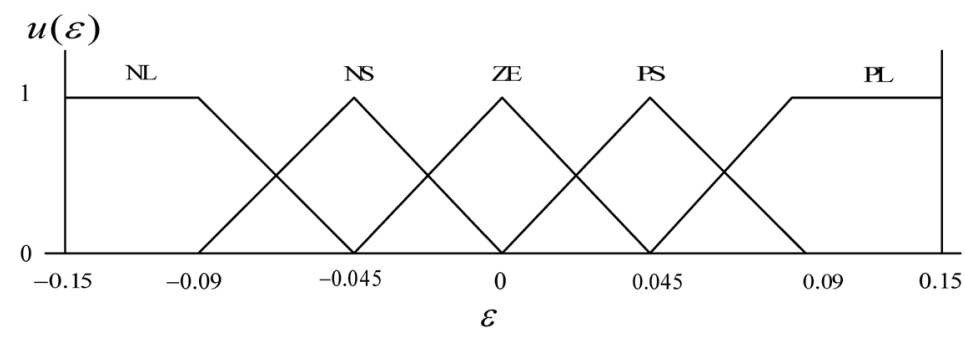

Fig. 6. Membership function of $\varepsilon$.

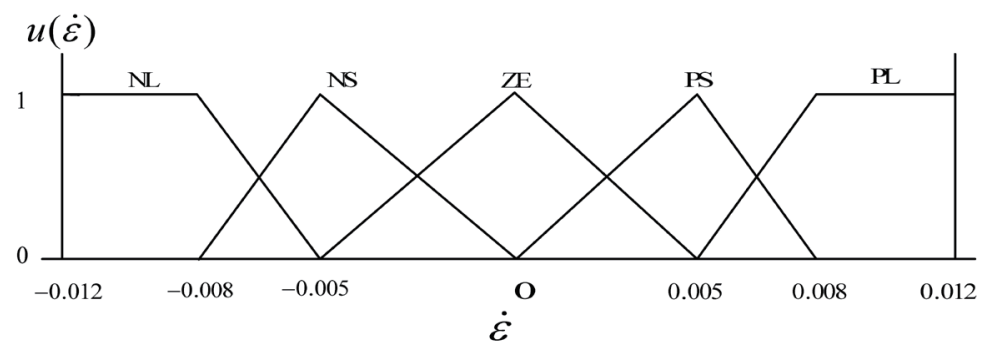

Fig. 7. Membership function of $\dot{\varepsilon}$.

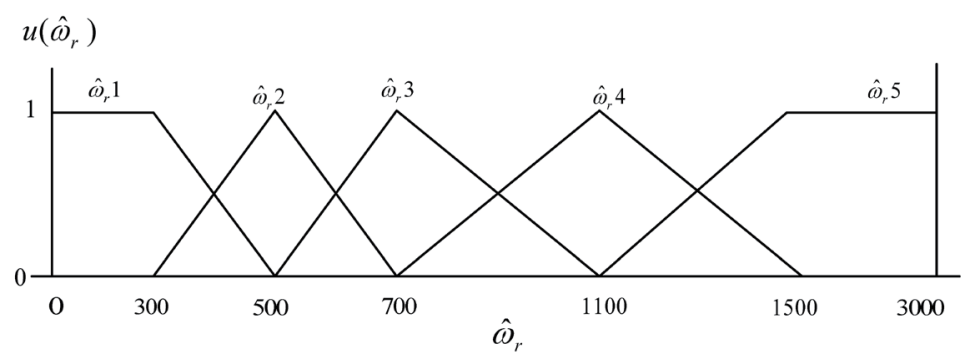

Fig. 8. Membership function of $\hat{\omega}_{r}$. 


\subsubsection{Fuzzy inference}

The output feature is decided by the fuzzy rule, and the output measure is dependent on the fuzzy inference. The Min-Min-Max method is used to determine the fuzzy inference. The first Min term is regarded as the fuzzification step and uses the minimum trigger as the membership grade. The second Min term is regarded as the output membership grade of each fuzzy inference rule, which selects the minimum value from the two input membership grades according to the fuzzy inference rules. The Max term is regarded as taking the maximum value, which integrates the same output membership functions into an individual rule. ${ }^{(21)}$

\subsubsection{Fuzzy rule}

The fuzzy rule is used to determine the trigger of the output membership function. In this system, the linguistic term "if-then" was selected as the fuzzy rule. The relationships between the input variables $\varepsilon$ and $\dot{\varepsilon}$ and the output variable $\hat{\omega}_{r}$ are shown in Table 2 .

\subsubsection{Defuzzification}

Defuzzification converts the inferred fuzzy output value into a crisp output value and uses this crisp output value to determine the estimated rotor speed. In this system, the central value of the defuzzification sum was used ${ }^{(21)}$ and defined as

$$
x^{*}=\frac{\sum_{l=1}^{m} q_{l} N\left(C_{l}^{\prime}\right)}{\sum_{l=1}^{m} N\left(C_{l}^{\prime}\right)},
$$

where $N\left(C_{l}^{\prime}\right)$ is the height of each $C_{l}^{\prime}$ and $q_{l}$ is the most central value of $x$ before the top of $C_{l}^{\prime}$ is cut off.

The block diagram of the proposed speed estimation adaptive DSFOC IM drive using the FLC strategy MRAS stator flux estimator is shown in Fig. 9. The system includes the following: speed controller, flux controller, $q$-axis and $d$-axis stator current controllers, $d$-axis

Table 2

Fuzzy rule table.

\begin{tabular}{|c|c|c|c|c|c|}
\hline$\dot{\varepsilon} \varepsilon$ & NL & NS & ZE & PS & PL \\
\hline NL & $\hat{\omega}_{r} 1$ & $\hat{\omega}_{r} 1$ & $\hat{\omega}_{r} 2$ & $\hat{\omega}_{r} 2$ & $\hat{\omega}_{r} 3$ \\
\hline NS & $\hat{\omega}_{r} 1$ & $\hat{\omega}_{r} 2$ & $\hat{\omega}_{r} 2$ & $\hat{\omega}_{r} 3$ & $\hat{\omega}_{r} 4$ \\
\hline ZE & $\hat{\omega}_{r} 2$ & $\hat{\omega}_{r} 2$ & $\hat{\omega}_{r} 3$ & $\hat{\omega}_{r} 4$ & $\hat{\omega}_{r} 4$ \\
\hline PS & $\hat{\omega}_{r} 2$ & $\hat{\omega}_{r} 3$ & $\hat{\omega}_{r} 4$ & $\hat{\omega}_{r} 4$ & $\hat{\omega}_{r} 5$ \\
\hline PL & $\hat{\omega}_{r} 3$ & $\hat{\omega}_{r} 4$ & $\hat{\omega}_{r} 4$ & $\hat{\omega}_{r} 5$ & $\hat{\omega}_{r} 5$ \\
\hline
\end{tabular}




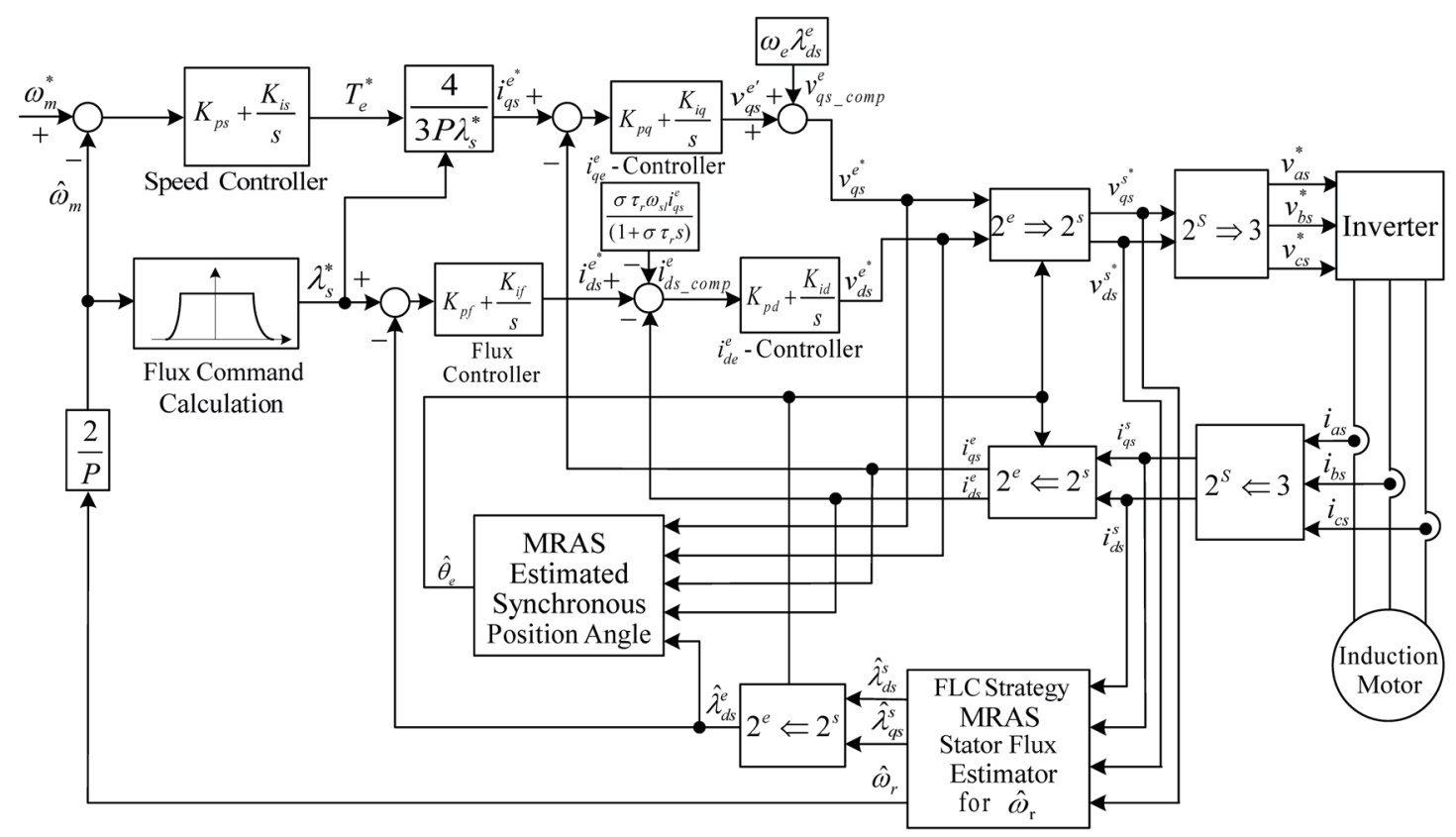

Fig. 9. Speed estimation adaptive DSFOC IM drive using the FLC strategy MRAS stator flux estimator.

stator current feed-forward compensation, $q$-axis stator voltage feed-forward compensation, flux command calculation, MRAS estimated synchronous position angle calculation, 2-axis synchronous frame to 2 -axis stationary frame coordinate transformation $\left(2^{e} \Rightarrow 2^{s}\right)$, 2-axis stationary frame to 2 -axis synchronous frame coordinate transformation $\left(2^{e} \Leftarrow 2^{s}\right)$, 2-axis stationary frame to 3 -phase system coordinate transformation $\left(2^{s} \Rightarrow 3\right), 3$-phase system to 2-axis stationary frame coordinate transformation $\left(2^{s} \Leftarrow 3\right)$, and the MRAS estimated rotor speed using the FLC strategy.

\section{Simulation and Experiment}

A standard 3-phase, $220 \mathrm{~V}, 0.75 \mathrm{~kW}, \Delta$-connected, squirrel-cage IM was used in the experiments to confirm the effectiveness of the proposed speed estimation adaptive DSFOC IM drive using the FLC strategy MRAS stator flux estimator. In a running cycle, the speed command was as follows: forward direction acceleration from $t=0$ to $t=1 \mathrm{~s}$; forward direction steady-state operation over $1 \leq t \leq 2 \mathrm{~s}$; forward direction braking operation to reach zero speed in the interval $2 \leq t \leq 3 \mathrm{~s}$; reverse direction acceleration from $t=3$ to $t=4 \mathrm{~s}$; reverse direction steady-state operation over $4 \leq t \leq 5 \mathrm{~s}$; and reverse direction braking operation to reach zero speed in the interval $5 \leq t \leq 6 \mathrm{~s}$. The simulated and measured responses, which include (a) command (dotted line) and actual (solid line) shaft speed, (b) command (dotted line) and estimated (solid line) shaft speed, (c) electromagnetic torque, and (d) stator flux linkage locus ( $q$-axis vs $d$-axis), are shown in Figs. 10-13. The simulated and measured responses for reversible steady-state speed commands of \pm 400 and $\pm 1800 \mathrm{r} / \mathrm{min}$ are shown in Figs. 10 and 11, and 12 and 13 , respectively. 


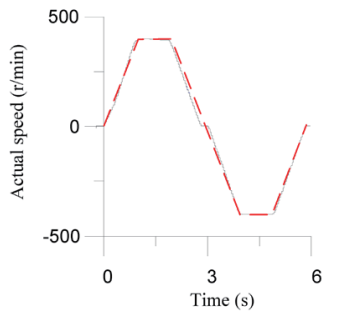

(a)

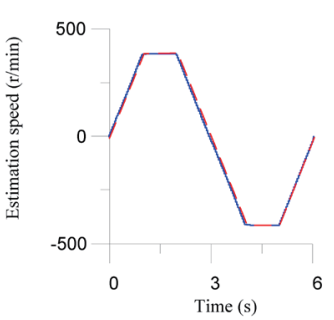

(b)

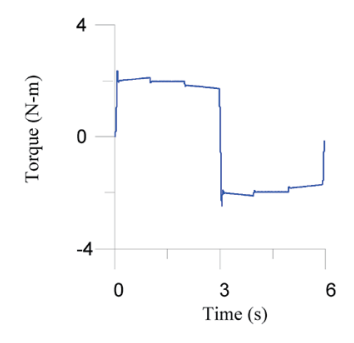

(c)

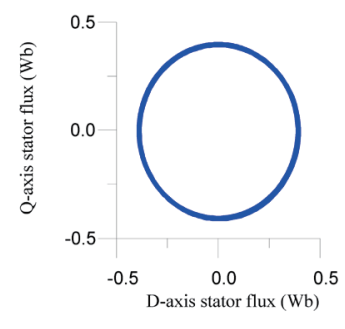

(d)

Fig. 10. (Color online) Simulated responses of the proposed speed estimation adaptive DSFOC IM drive using the FLC strategy MRAS stator flux estimator at the reversible steady-state speed command of $\pm 400 \mathrm{r} / \mathrm{min}$ : (a) actual shaft speed, (b) estimated shaft speed, (c) electromagnetic torque, and (d) stator flux linkage locus.

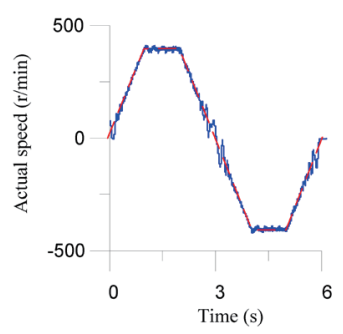

(a)

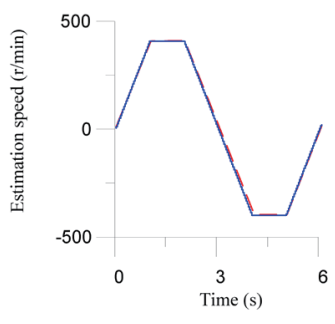

(b)

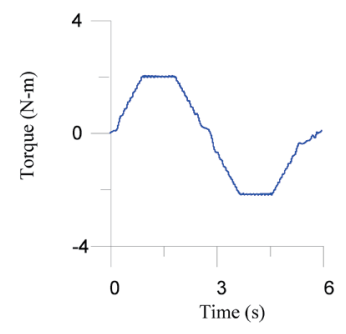

(c)

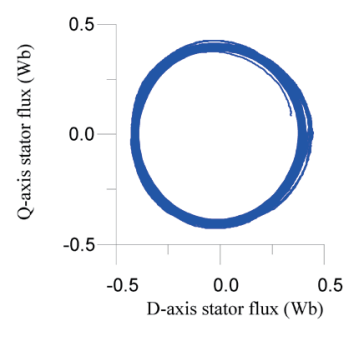

(d)

Fig. 11. (Color online) Measured responses of the proposed speed estimation adaptive DSFOC IM drive using the FLC strategy MRAS stator flux estimator at the reversible steady-state speed command of $\pm 400 \mathrm{r} / \mathrm{min}$ : (a) actual shaft speed, (b) estimated shaft speed, (c) electromagnetic torque, and (d) stator flux linkage locus.

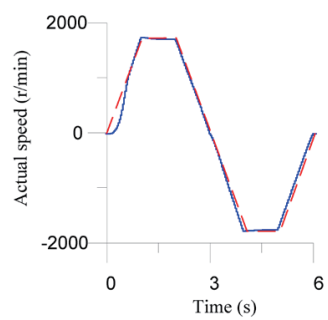

(a)

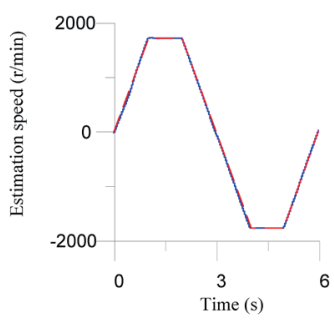

(b)

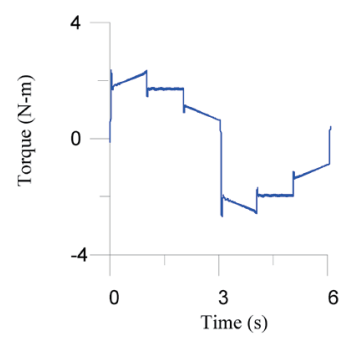

(c)

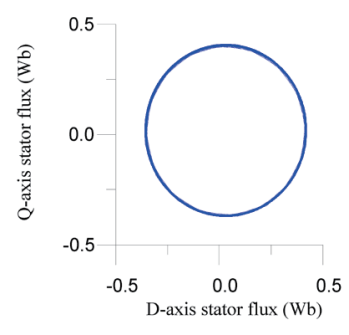

(d)

Fig. 12. (Color online) Simulated responses of the proposed speed estimation adaptive DSFOC IM drive using the FLC strategy MRAS stator flux estimator at the reversible steady-state speed command of $\pm 1800 \mathrm{r} / \mathrm{min}$ : (a) actual shaft speed, (b) estimated shaft speed, (c) electromagnetic torque, and (d) stator flux linkage locus.

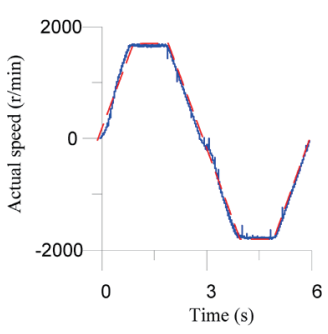

(a)

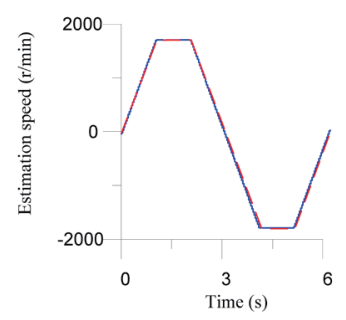

(b)

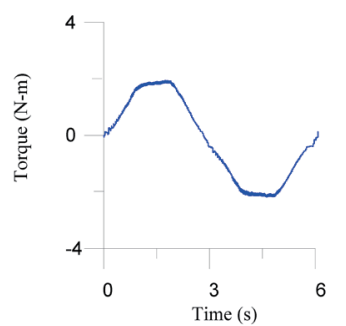

(c)

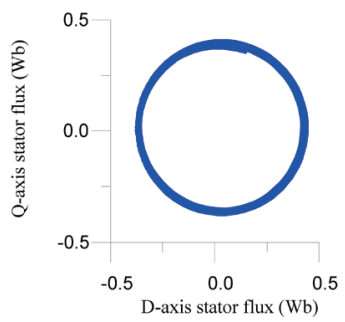

(d)

Fig. 13. (Color online) Measured responses of the proposed speed estimation adaptive DSFOC IM drive using the FLC strategy MRAS stator flux estimator at the reversible steady-state speed command of $\pm 1800 \mathrm{r} / \mathrm{min}$ : (a) actual shaft speed, (b) estimated shaft speed, (c) electromagnetic torque, and (d) stator flux linkage locus. 
On the basis of the simulated and measured results for different operational speeds shown in Figs. 10-13, the accurately estimated rotor-shaft speed was attained, the electromagnetic torque achieved superior responses, and the estimated circular stator flux locus guaranteed that the coordinate transformation between the synchronous and stationary reference coordinate frames is exact. The proposed speed estimation adaptive DSFOC IM drive using the FLC strategy MRAS stator flux estimator has shown that the desired performance can be attained.

\section{Conclusion}

The adaptive stator flux estimator and the synchronous position angle identification scheme were developed to establish a speed estimation adaptive DSFOC IM drive. The MRAS stator flux estimator, according to the FLC strategy adaptation mechanism, was used to acquire an accurately estimated rotor-shaft speed. The MRAS synchronous position angle identification scheme based on the reactive power was utilized to guarantee that the coordinate transformation between the synchronous and stationary reference coordinate frames is exact. The stator current signal measurement for implementing the DSFOC IM drive and the adaptive stator flux estimator was provided by the Hall effect current sensors. The simulated and measured responses (including the estimated rotor speed, electromagnetic torque, and stator flux locus) at two different reversible steady-state speed commands ( \pm 400 and $\pm 1800 \mathrm{rpm}$ ) confirmed the effectiveness of the proposed approach.

\section{References}

1 Y. C. Luo, C. L. Tsai, and Y. P. Kuo: Sens. Mater. 30 (2018) 845. https://doi.org/10.18494/SAM.2018.1788

2 M. Montanari, S. M. Peresada, C. Rossi, and A. Tilli: IEEE Trans. Cont. Syst. Tech. 15 (2007) 1049. https:// doi.org/10.1109/TCST.2007.899714

3 M. Kumar and S. Das: IET Pow. Elect. 11 (2018) 2355. https://doi.org/10.1049/iet-pel.2018.5344

4 K. Ide, J. L. Ha, and M. Sawamura: IEEE Trans. Ind. Elect. 53 (2006) 130. https://doi.org/10.1109/ TIE.2005.862306

5 Z. M. Elbarbary, H. A. Hamed, and E. E. El-Kholy: IEEE Trans. Pow. Elect. 33 (2018) 8187. https://doi. org/10.1109/TPEL.2017.2743681

6 F. M. Fayez and E. I. Sousy: IEEE Trans. Ind. Info. 9 (2013) 1922. https://doi.org/10.1109/TII.2013.2238546

7 R. J. Wai: IEEE Trans. Ind. Elect. 54 (2007) 586. https://doi.org/10.1109/TIE.2006.888807

8 R. J. Wai and H. H. Chang: IEEE Trans. Neur. Netw. 15 (2004) 367. https://doi.org/10.1109/TNN.2004.824411

9 I. Boldea, M. C. Paicu, and G. D. Andreescu: IEEE Trans. Pow. Elect. 23 (2008) 2612. https://doi.org/10.1109/ TPEL.2008.2002394

10 C. Korlinchak and M. Comanescu: IET Elec. Pow. Appl. 6 (2012) 353. https://doi.org/10.1049/iet-epa.2011.0245

11 E. D. Mitronikas and A. N. Safacas: IEEE Trans. Ind. Elect. 52 (2005) 1660. https://doi.org/10.1109/ TIE.2005.858706

12 M. Jemli, H. B. Azza, M. Boussak, and M. Gossa: IEEE Trans. Pow. Elect. 24 (2009) 1618. https://doi. org/10.1109/TPEL.2009.2014867

13 M. Farasat, A. M. Trzynadlowski, and M. S. Fadali: IET Elec. Syst. Transp. 4 (2014) 122. https://doi. org/10.1049/iet-est.2014.0018

14 C. A. Reusser, C. A. Silva, and P. Dominguez: Proc. IEEE Ind. Elect. Soci. (2013) 4874. https://doi.org/10.1109/ IECON.2013.6699924

15 S. Xingfa, N. Ziling, Z. Junjie, H. Yi, Y. Weiwei, S. Jun, and W. Yanhao: Proc. IET Airc. Util. Syst. (2018) 769. https://doi.org/10.1049/cp.2018.0136

16 C. H. Liu: Control of AC Electrical Machines (Tunghua, Taipei, 2008) [in Chinese].

17 B. Shafai: System Identification and Adaptive Control (Springer Verlag, Switzerland AG, 2014). 
18 Y. C. Luo and Y. H. Chen: Intel. Fuzz. Syst. 36 (2019) 1215. https://doi.org/10.3233/JIFS-169895

19 Y. C. Luo and W. X. Chen: Comp. Math. Appl. 64 (2012) 1206. https://doi.org/10.1016/j.camwa.2012.03.064

20 Y. C. Luo, Y. H. Chen, and Y. P. Kuo: Sens. Mater. 31 (2019) 165. https://doi.org/10.18494/SAM.2019.2020

21 Y. C. Luo and C. L. Tsai: Low Freq. Noise. Vibr. Act. Contol 38 (2019) 1220. https://doi. org/10.1177/1461348418784187

\section{About the Authors}

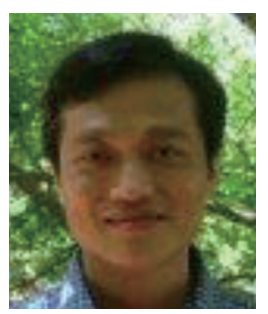

Yung-Chang Luo received his M.S. and Ph.D. degrees from National Taiwan University of Science and Technology, Taipei, Taiwan, ROC, in 1991 and 2000, respectively. He is currently a professor in the Department of Electrical Engineering, National Chin Yi University of Technology, Taichung, Taiwan, ROC. His current research interests include speed estimation ac motor drives, the front-end power factor correction of converter, and the design of microcontroller-based motor drive.

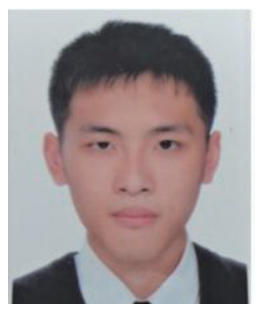

Bo-Wei Chen is a master student in the Department of Electrical Engineering, National Chin-Yi University Technology, Taiwan. His main research focus is on speed estimation field orientation controlled induction motor drives.

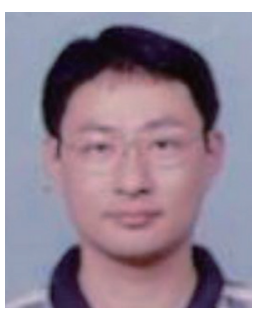

Wen-Cheng Pu received his Ph.D. degree from National Taiwan University of Science and Technology, Taipei, Taiwan, ROC, in 2010. He is currently an associate professor in the Department of Electrical Engineering, National Chin Yi University of Technology, Taichung, Taiwan, ROC. His current research interests include the speed estimation of ac motor drives, automatic technology, and the design of microcontroller-based motor drive.

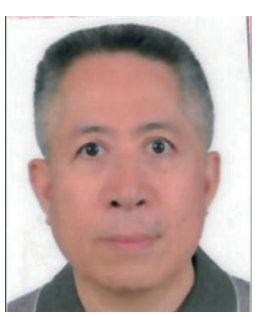

Neng-Sheng Pai received his B.S. and M.S. degrees from the Department of Automatic Control Engineering, Feng Chia University, Taichung, Taiwan, ROC, in 1983 and 1986, respectively. In 2002, he received his PhD degree from the Department of Electrical Engineering, National Cheng Kung University, Tainan, Taiwan, ROC. He is currently a professor in the Department of Electrical Engineering, National Chin-Yi University of Technology, Taichung, Taiwan, ROC. He was the chairman of the department from 2004 to 2007 and was also the chairman of the Computer Center of National Chin-Yi University of Technology from 2013 to 2017. His current research interests include fuzzy systems, artificial intelligence, imagine processing, advanced control systems, and microprocessor systems. 